\title{
Choice-making and choose-ables: making decision agents more human and choosy
}

\section{Lorraine Dodd $^{1} \mathbb{D}$}

Received: 22 September 2017 / Accepted: 6 December 2018 / Published online: 14 December 2018

(c) The Author(s) 2018

\begin{abstract}
This paper discusses concepts that might shape, extend, limit or re-focus an agent's set of options that can then be thought of as that particular agent's potential in terms of their ways forward and degrees of freedom. Because there is no unambiguous word that conveys the meaning of this higher order concept of choice-making, the term "choose-able" has been adopted in order to distinguish it from the usual decision concepts known as choice or option. An agent's choose-ables are defined as the imagined deemed possible ways forward, that the agent has to construct, compose or create before they can choose. The central concept of a choose-able is a very powerful one if only it could be surfaced and made explicit. It is often only possible to make inferences about the nature of choose-ables after observing the actions taken once a choice has been made. Drama theory formally develops this kind of inferencing and provided a foundation for this paper as it explores the relational realms of options. The paper presents a funnelling construct and then draws together Catastrophe theory and Culture theory to offer new ways of analysing the shaping effects of relational contexts on an agent's choose-ables that then act as a medium through which agents are drawn to make choices and carry out observable actions. The strength of the combination of the theories lies in their descriptive power of subjective, relational concepts that hitherto have tended to remain hidden and tacit.
\end{abstract}

Keywords Decision options $\cdot$ Choice $\cdot$ Catastrophe theory $\cdot$ Culture theory

Mathematics Subject Classification 91C99 - 91B14

Lorraine Dodd

L.dodd@cranfield.ac.uk

1 Decision and Information Management Group, Cranfield University, Defence Academy of the UK, Room MH103b, Shrivenham, Wiltshire SN6 8LA, UK 


\section{Introduction to choice-making and choose-ables}

The subject of agent-based decision-making spans many areas of research, simulation and modelling; as covered in the recent comprehensive survey by Balke and Gilbert (2014) that compares and contrasts architectural frameworks for modelling decision agent processes. These agent-based decision frameworks deal principally with modelling an agent's course of action selection from a given set of options. This paper does not aim to extend those existing architectural frameworks for selection of "a choice" from a set of options. ${ }^{1}$ This paper deals with the contextual factors involved in composing an agent's set of options in the first place.

This paper discusses concepts that might shape, extend, limit or re-focus an agent's set of options that can then be thought of as that particular agent's potential in terms of their ways forward and degrees of freedom. Because there is no unambiguous word that conveys the meaning of this higher order concept of optionmaking or choice-making, ${ }^{2}$ the term "choose-able" has been adopted in order to distinguish it from the usual decision concepts known as choice or option. The term "choose-able" is introduced because there does not seem to be a formal name for the concept of a choose-able. It has been adopted from work by Shackle (1976): "Your list of choosable things has to be constructed or composed by yourself before you can choose“. An agent's choose-ables are defined as the imagined deemed possible ways forward that the agent has to construct, compose or create before they can choose.

It is an exploration of why a decision agent's circumstances and relational conditioning might shape or directly affect their choose-ables. The central question being addressed is: what might be intrinsically or extrinsically affecting an agent's degree of decision freedom? So this paper is about understanding conditions for the shaping of choose-ables, from which decisive consequences may (or may not) then flow. It is trying to reflect a more analogue approach to thinking about how an agent's potential for choice might be scoped or broadened. The approach works through from contextual, subjective conditions to consequential potential, via a notional 'funnelling' that works to either limit or open-up an agent's choose-ables.

Choose-ables cover the possible ways forward that any agent:

- Can conceive of (or imagine).

- Would countenance as being one of their choose-ables (e.g. within their moral code $\left.^{3}\right)$.

- Feel are open to them for choice (e.g. given their relational circumstances).

- Feel they are obliged to consider.

\footnotetext{
1 The paper remains agnostic about operational feasibility and desirability at the time of option selection, which tends to be the usual focus of agent decision-making architectures.

${ }^{2}$ Choice-making is used here to differentiate it from decision-making and decision-taking. This paper is not about rationales for decision-taking, which lie in the realms of Decision theory and Game theory and where there tends to be a pre-defined set of options; each with a likelihood of outcome or pay-off.

3 There are deeper philosophical foundations that underlie much of this paper (Arendt 1982).
} 
- Feel competent to consider.

- Feel they are barred from considering (e.g. social taboos).

There are strong similarities with the concept of affordance (Bradshaw et al. 2004a) in that choose-ables describe the subjective contextual nature of the opportunities for choice-making and the 'wiggle room' for consideration of potential ways forward. Affordances are properties of the context taken relative to any person or agent (Wells 2002) and as such they do not explicitly define that agent's constraints or desires, being more about perception of environment ${ }^{4}$ inviting an active response. Affordances pertain to the context but are relative to and subject to what that context offers, opens up or closes off, perceptually as well as operationally (Gibson 1979), for that decision agent in their current personal circumstances. This does not explicitly include the agent's capabilities in terms of what might be predicted to be probable outcomes of decisions, ${ }^{5}$ both of which will further affect an agent's ultimate choice of option. An agent's appreciation of how difficult or impossible it is to predict is related to their ability to look at context. ${ }^{6}$ That difficulty or impossibility to predict tends to increase with complexity and intricacy of context.

In essence, the paper proposes conceptual extensions into higher orders of decision agility (Dodd and Markham 2013) and higher levels of adaptation (Grisogono 2004) to reason about an agent's potential or degrees of freedom for choice. The proposed conceptual frameworks are underpinned by developmental theories drawn from the mathematics of discontinuity (Zeeman 1977), subjective economics (Shackle 1976) and from Culture theory studies (Douglas 2008). The aim is to address how levels of adaptation might be extended within decision agent frameworks to capture how and why agents might limit or extend their choose-ables (as listed previously).

This paper provides a framework for reasoning why agents might be subjectively scoping their focused set of options and how the ever-changing context is working to shape their choose-ables. So it offers a way to address (and maybe eventually to encode), as a whole, what might subjectively scope, shape, constrain, restrain, openup, extend or enhance an agent's choose-ables.

The nearest there is to a collective concept might be referred to as an agent's portfolio of choices that are deemed to be OK by them and for them to choose from. The important point being that the nature of that deeming of 'OK-ness' is temporally subjective; that is, according to that agent (or agency) at that time and in terms of their particular circumstances and concept of time span. This collective concept can also relate to a person, group or an agency (Sylvan and Voss 1998): "Previous studies of foreign policy decision making have largely focused on the choice among specified options rather than the prior question of how the options were specified in the first place."

\footnotetext{
${ }^{4}$ For more detail see Klugl (2015).

5 In terms of 'Ways, Means, Ends' the focus here is firmly on the scope and nature of the ways forward and is not directly concerned with the available means or the desired operational ends.

${ }^{6}$ Referred to by Harvard's Nohria, Mayo and Khanna as "contextual intelligence" (see Khanna 2014).
} 
This requires a step back to explore what might be happening in that agent's relational, cultural, 'personal', organizational and temporal contexts. This further requires access to an agent's implicit, trust-based, rationale for what they might (or might not) be open to considering as one of their choose-ables, given their current circumstances and 'personal' experience to date. For example, a vegetarian 'agent', in their everyday circumstances, would not countenance having "eat meat" as one of their choose-ables; however, if their circumstance was one of extreme hunger and they were being kindly and generously offered meat stew, then they may extend the scope of their choose-ables temporarily to include "eat meat". The strength of their cultural relationships and personal positioning plays a large part too, as described by Douglas (2008). For example, if they are tied-in to a patriarchal grid structure or if they choose to conform to the values of a social group then their degree of autonomy in choice-making will be relatively shaped or biased.

Choose-ables are, therefore, subjective and personal and tend to be shaped, limited and/or opened-up according not only to an agent's context but also to the extent and nature of their awareness of that context. This contextual framing can also extend the concept of a choose-able to be not only about active ways forward (e.g. eating meat), but also ways of making sense of situations (e.g. interpreting a scene as $\mathrm{A}$ or B), of where to place their attention (e.g. focused or wide attention ${ }^{7}$ ), or what to believe, whose preferences to take account of, etc. This can be extended further if we are to consider decision agents (e.g. agents representing managers) whose choose-ables might be organisational (e.g. empowerment of others) or ways to shape relationships (e.g. building of governance relationships).

The basis for a previous "Theory of Choice" paper (Dodd 2011) is that chooseables tend to frame the ways in which agents may be able to sense, feel, interpret, discern, assess, adapt and act in their world (Dodd et al. 2008). In turn, all of these are shaped and conditioned by the nature and scope of the ways forward that appear to be open to them at the time of need for choice-making and decision-making. For example, if an agent has only one way forward, then the situation may need to be seen and framed to fit that one way forward to provide the comfort of knowing and of having "the solution to the problem"; as in: if I only have a hammer then every situation for me becomes a nail to be struck. For example, western governments, bound by accountability and strict legal scrutiny, are often limited to only one choose-able, such as imposing sanctions. Their focus on sanctions then tends to limit the way in which they see and make sense of the situation. This naturally suggests that the influences flow both ways between the sensing or sense-making and the choose-ables that are seemingly open for choice; therefore, having a closed mind and firmly focused eyes tends to close-off options for choice; however, not being open to more options for choice tends towards finding comfort in one's closed mind and ears and focused eyes.

\footnotetext{
7 "The second way of perceiving seemed to occur when the questioning purposes were held in leash. Then, since one wanted nothing, there was no need to select one item to look at rather than another, so it became possible to look at the whole at once." (Milner 1986, p 106).
} 
The central concept of a choose-able is a very powerful one if it can be surfaced and made explicit. The other related concept is, as previously mentioned, "affordance", which is taken here to be a relation between a context (of agency and people) and an agent (Bradshaw 2003) that affords the opportunity for that agent to perform a decisive action. The idea of affordance is relatively new ${ }^{8}$ and it is essentially a concept of design, or policy, giving way (i.e. offering a way forward) to subjective choice. The idea of affordance has been developed further, as a relation between an agent and its context, in the domain of human-machine cognition, as in Bradshaw et al. (2004a). Figure 1 depicts the relationship between the extent of an agent's imaginable 'wiggle room' for choice ${ }^{9}$ and that bounded by a policy-based structure or design; the relevant area here is the area marked "wiggling the wiggle-room" as this is a simple representation of the imagined deemed possible (i.e. choose-ables).

These intangible, relational concepts are difficult to make explicit. Agents (also agencies and people) do not openly display or make explicit their choose-ables and they may not even be explicitly aware of them. It is only possible to make inferences about the nature of choose-ables after observing the actions taken once a choice has been made. For instance, if we see a person carrying out an extreme form of action, then we could infer from that chosen action that their choose-ables extend further than the scope of the majority. Drama theory ${ }^{10}$ (Bennett et al. 2001) formally develops this kind of inferencing and provides a foundation for this paper as it begins to explore the relational realms of decision options. Drama theory helps analysts to reason about the contextual shaping of a decision agent's openness and willingness to imagine, countenance or consider an option for choice in order that it may then be deemed worthy (or not) of becoming one of their choices for action selection, ${ }^{11}$ finally resulting in observed behaviour.

\section{Extending agent frameworks: capturing the context}

Shackle (1976) introduced the concept of choose-ables in terms of: "the imagined, deemed possible". His mathematical notation is unusually subjective, which makes for a challenging read for those who require " $x$ " to be an objective variable. Shackle's three functions represent personal preference, belief and focus (i.e. of attention or interest). These functions are composed to shape decision conditions

\footnotetext{
8 James Gibson (see Gibson (1977)) and Donald Norman (see Norman (1999)) are two of the main originators of the idea. As an example, a door with a pull handle would suggest that it should be pulled and not pushed, so the handle holds an affordance for pulling.

9 “An agent's 'wiggle room' consists of its set of performable actions in a given context, while the policy-based bounds that people impose on that wiggle room define a smaller region of trusted operation. Capabilities for adjustable autonomy support the modification of these bounds at runtime in order to adapt to changing conditions." Bradshaw et al. (2004b).

10 Drama theory is referred to here rather than "Confrontation Analysis" as Drama theory opens up imagination and inquiry about possible and plausible hidden subjective motives and (often unknowable) relational states.

11 These are referred to as "cards" in Confrontation Analysis (see Howard 1999).
} 


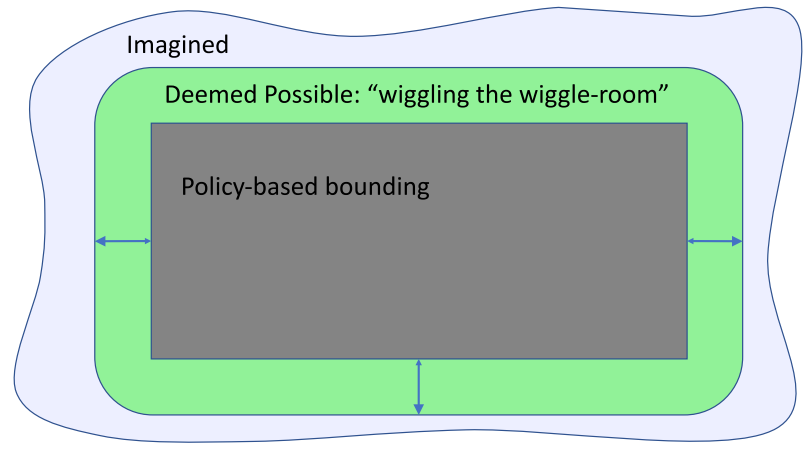

Fig. 1 An agent wiggling its wiggle room (Bradshaw et al. 2004b)

and consequences, where time is not constrained to be objectively chronological (i.e. chronos time) but can be interpreted as being of subjective opportunity [i.e. timing or kairos time in Dodd and Markham (2012)].

This exploration of Shackle's (1976) concept of choose-ables proposes a practical construct, which can then be abstracted into frameworks that refer to non-linear mathematics and Culture theory, to capture the context in relation to subjective choice-making.

The proposed practical constructs are based on a triangular foundation that asks:

What might be shaping an agent's choose-ables?

Essentially there is a sense of 'three-ness' running through the narrative of this paper. Shackle's three integrated functions of belief, preference and focus relate more to an agent's sensing and sense-making for choice-making. There are other foundational theories that are triangular in nature, yet relate more to choice-making for action-taking: either for active, decisive or adaptive action.

Figure 2 gives a representation of Turing's association of the concepts of (1) drive (or motivation) and (2) need for order, with (3) a variety of stable options. If an agent's adherence to policy-based systems of order (e.g. laws or norms) is strong and their drive to act differently is low, then their variety of options will tend to be relatively small. Turing's foundational trinity is more in the spirit of morphogenesis ${ }^{12}$ as it relates to an on-going 'bandwidth' of adaptive capability; however, Turing's conceptualisation of variety of options for choice as a bandwidth of adaptive capability is relevant to the concept of 'wiggle-room' in terms of extended degrees of affordance, as discussed previously.

Similarly, Clausewitz's trinity in his book “On War" [first published in 1832 but see Clausewitz (1873)] captures an agent's freedom for choice being dependent on (1) an agent's drive towards action; (2) an agent's subordination to instruments of policy or power; and (3) an agent's relationship with context. The Clausewitz trinity is often interpreted simply as the three terms: agency, governance and action;

12 Interpreted from discussions with Dr. David Marsay on variety of stable states based around Turing (1952). 


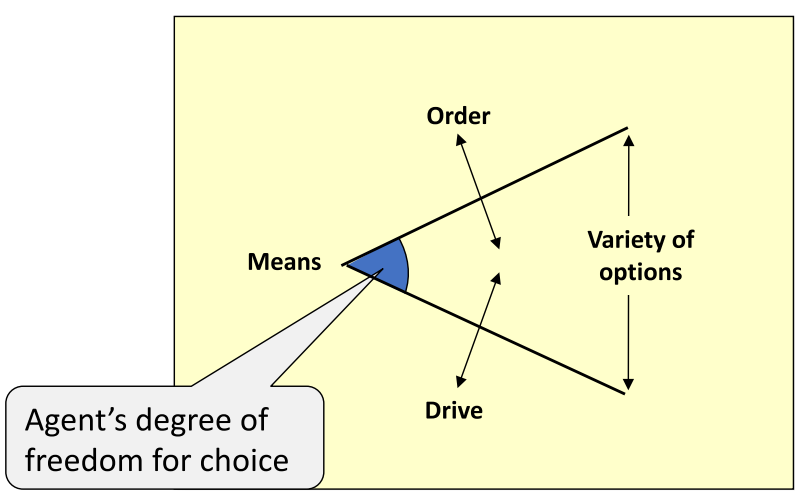

Fig. 2 Agent's drive and adherence to order relating to variety of options

however, Clausewitz described his trinity as an interactive set of three basic dominant tendencies that drive the events of war, composed of: "primordial violence, hatred, and hostility; its element of subordination as an instrument of policy; and the play of chance within which the creative spirit is free to roam." This Clausewitzian view suggests that the choose-ables represent choice-based 'ends' in the form of aiming for an inherent active capability; for instance, we can then say that if a person is given a deadly weapon and the training to use it, also is bound by subordination to political orders, and has the will to use the weapon in specific relational contexts, then a person is capable of political killing.

The practical funnelling construct holds that choose-ables can be related to capacities or competences, moderated by will and motivation (e.g. the highly motivated agents are more likely to adapt and extend their choose-ables) and the degree to which they might be in accordance with policy, rules and regulatory laws (i.e. that are felt necessary to abide by). The broadening or narrowing of the funnel (see Fig. 3) represents the opening-up, closing-off, trading-in or trading-out of chooseables and begins to bring to light the potential openings and constraints due to the relational context of the agent (i.e. in terms of structural couplings). The graded colouring from blue to green within the funnelling construct refers to the agent's "deeming" of the imagined to the possible.

A useful question regarding the contextual state of an agent could be: "Where might the agent stand, relationally speaking?" (where this includes their position relative to power and authority, for example). This could be followed by: "What might the agent be trying to achieve? and what might they believe they need to do (and might be capable of doing or not doing) to achieve that?" Many assumptions will have to be made to answer these questions; however, a useful indicator that our assumptions may be open to further question would be if something arouses surprise.

It is the nature of the relationship between the context and the agent that is important. If an agent is socially open to working with others' preferences and considering others' perspectives, then choose-ables will be more open to including others' 


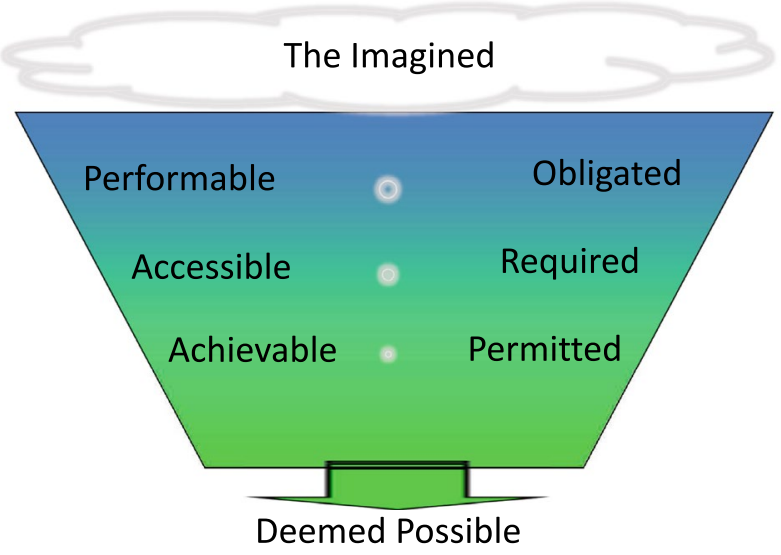

Fig. 3 A triangular funnelling construct to represent shaping of choose-ables (adapted after Bradshaw et al. 2004a)

options and also open to finding compromises that are OK for all concerned. ${ }^{13}$ The agents' choice-making then is more about finding a resolution that is acceptable to all concerned ${ }^{14}$ than finding a solution that is 'best' according to the agent's preference.

This also applies to people in today's society, with ever tightening norms and demands to deliver targets. Choose-ables are often traded-out due to restraints and constraints imposed by the institutional or organizational context. This, though degrees of contextual intelligence, may then be tempered by one's outlook to the wider scope of relationships and/or immediate needs. For example, one may be tempted to consider including a choose-able that goes outside the norms or rules, if it could open up useful relationships. The scope of choose-ables can often be most severely constrained by institutional norms and rules; so there is conformance and compliance to keep to those choose-ables that are consistent and 'normal'. The Greek term phronesis, or practical wisdom, can be described as knowing when to judge which rules to break and by how far.

This mix of contextual and textual constraints and restraints provides a rich texture $^{15}$ through which to study what is happening at the boundaries between the context and the text of the choose-ables. It is across this boundary that the text describing the set of choose-ables is shaped by the context, through an agent trading-in or trading-out their choose-ables (according to the changing context, which might be

\footnotetext{
13 These can be categorised (as at University of Groningen http://www.rug.nl/feb/education/complexity -uncertainty) as being normative, conformative, non-conformative, and anti-conformative that then drive emergent patterns of behaviour; i.e. an agent in those interactive categories can generate particular system patterns. Some can produce institutional 'lock-in', resilient to change, when only drastic changes in circumstances may cause this lock-into be broken.

14 See Kant's notion of "enlarged mentality" in Arendt (1982).

15 Emery and Trist (1963) refer to this as a causal texture.
} 
a kind of dynamic conditional landscape that shapes consequential actions through a medium of choose-ables). A helpful matrix is provided in Christensson (2008) that works through three staged transformations to address the how, what and why actions may be considered in a collective context.

There is also the notion of subtext, which is covered in more depth in Drama theory and Confrontation Analysis, when an actor's choose-ables are left openly 'on the table' (or can be left hanging ambiguously) in order to threaten, coerce and persuade others. For example: "We are keeping the option of nuclear strike on the table". This aspect of subtext will not be covered here as the focus is on general context for shaping choose-ables rather than what can be consciously used to undermine or influence others' choices; although there are strong links between the two.

\section{Bringing context to bear through Culture theory}

Culture theory offers a relational view of agents in context. Thompson (2008), based on Douglas (2008), suggests four relational states (see Fig. 4): hierarchist, individualist, egalitarian and fatalist. Each appears to have a different characteristic choice function, which could be used to represent an agent's choice-making when in different relational contexts. For instance, when in a hierarchist state the agent tends to subscribe strongly to group norms and also to a defined grid structure (e.g. a military culture) so tends to be trying to find a workable balance across a hierarchy of purpose, aiming to take other agent's preferences and perspectives into account to find a balanced resolution; whereas when in the individualist state, the agent tends to work to their own preference and perspective, being only weakly held by formal structures (e.g. rules of law, ethics, the market place, etc.) and driven by their own OK-ness as the norm (e.g. making profit, survival of the fittest in the market place).

The assumption is that each relational context holds to a correspondingly different approach to choice-making [thence to decision risk (Douglas and Wildavsky

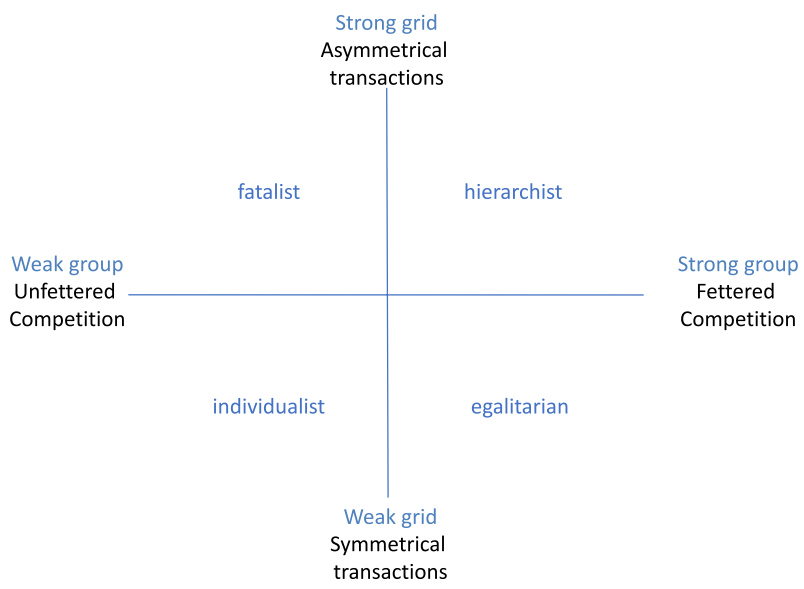

Fig. 4 Relational states from Thompson's depiction of Douglas' grid-group frame (Thompson 2008) 
1982; Thompson et al. 1990)] also the representations of choice embody the different natures of the agent's preference, belief and focus functions relating to being more or less attracted towards any of the four states. It is not so much that the four relational states are centres of attraction; it is more that the complex relational field or surface, which helps to differentiate between the four states, is acting to shape where an agent's socio-cultural relationships might be helping them to settle (i.e. at-traction) or making them feel a need to move away (i.e. negative e-motion). For example, put simply, a fatalist state of being has a more flattened preference function (i.e. there is no particular personal preference about potential consequences) and so choose-ables are more readily traded-in or traded-out (i.e. open to anything or not minding anyway). This can be compared with those corresponding to the other states, where there is more concern for one's own perspective and preferences and for appreciating and upholding those of others. As and when agents are drawn towards (or repulsed away from) any of the states, the ways in which they differentiate themselves from others or integrate themselves with others affects their choose-ables.

If agents are openly, and coherently, well-integrated into their social context, then they are more likely to consider being open to other's choose-ables as well as their own. ${ }^{16}$ If segregated, then they will focus on choose-ables that they know will maintain their own 'OK-ness'. The circumstances in which agents find themselves can have a strong bearing on the degree of autonomy and heteronomy that they are willing and able to accept (Smith and Dodd 2012). Thompson (1982) had added a power dimension to the grid-group model that would be necessary to consider in conditions where some agents assume positions of power in relation to other agents.

\section{Adding descriptive mathematics: a step towards modelling}

A more rigorous mathematical basis can be developed from the nature of variety in the different agents' principles of being that translate across into preferences for retaining choose-ables (and preferences for rejecting them). The mathematics expresses what might affect those preferences when circumstances present a need to resolve the principles of being, through trading-in or trading-out certain chooseables, as and when there might be a contextual need for survival, coexistence, collaboration and cooperation. It also captures important differences in an agent's principles of being. For example, some agents may be unaware of (or indifferent to) other agent's preferences and care only for their own ways of being.

The mathematical essence is drawn from Catastrophe theory (Zeeman 1977; Smith et al. 1981) that links four shaping coefficients ${ }^{17}$ relating to different relational

\footnotetext{
16 This would depend on whether or not they have a cohesive goal, as tight cohesion could result in adherence to goal-related task and 'group-think' confirmation. This is in contrast to being coherently aligned to a common purpose.

17 There are a further three elliptoid catastrophes. This paper refers only to the cuspoids.
} 
conditions and thence to different resultant choose-ables. The four shaping coefficients can be related to the following factors:

- Coefficient $a$ : Combination of critical 'normal' factors used to monitor and maintain an agent's autopoietic sense of 'OK-ness'. This sense of 'OK-ness' can then focus choose-ables down to adaptive thresholds that are known to provide success, viability, homeostasis and/or survival.

- Coefficient $b$ : Splitting 'confusion' factors that represent the degree of uncertainty, ambiguity or inconsistency being felt due to a situation being complex and/or pertaining to two or more conflicting perspectives. These factors can then be linked to the degree of certainty needed ${ }^{18}$ by the agent concerning the ongoing situation, state or status.

- Coefficient $c$ : Belief 'bias' factors used for seeing biases and differences in projected futures (e.g. short-term vs long-term views) and beliefs in own and/or other agents' ideas of possible/likely/plausible futures. This links choose-ables either firmly to one agent's belief 'system' or leaves them open to a variety of other agents' beliefs about how things might go. ${ }^{19}$

- Coefficient $d$ : Assessment 'value' factors for evaluating and resolving preferences and perspectives when appreciating own and other agent's preferences and perspectives. An agent's approach to finding an appreciative resolution can lead that agent towards creation of new choose-ables (e.g. finding middle ground, resolution or compromises) that can to be readily accommodated into own preferences and into other's worldviews and ways forward.

The important thing to note is that these four coefficients not only change over time, as relational circumstances change, also they become more or less relevant when functional needs and living conditions change (i.e. need to be working in harmony with others and within a balanced position in the working environment ${ }^{20}$ ).

The fold catastrophe has only the first coefficient, so is appropriate for describing an agent's choice-making when there is just the focused objective of its own targeted priority. Here, the choose-ables support only the focused utility of the immediate; for example, a financial trading agent according to factors contributing to coefficient $a$ crossing a threshold value.

The cusp catastrophe with coefficients $a$ and $b$ is the most familiar and is adequate to represent most agents' choice-making. For example, with sense of homeostatic thresholding being represented by coefficient $a$, and with degree of cognitive dissonance being represented by coefficient $b$ the agent's choice-making could be represented descriptively. The cusp region represents an area of bi-stability where the choose-ables become increasingly binary (e.g. fight or flight) due to drift in their

\footnotetext{
18 This can, in extreme cases, lead to a bi-polar situation where the system flips between one choose-able and the other.

19 See related ideas in Marsay (2015).

20 Angyal (1958) termed these two conditions to be homonomy and heteronomy, respectively.
} 
relational context, when tiny changes in conditions can result in a discontinuous shift.

A catastrophe equation with the first three coefficients is called a swallowtail catastrophe, where the choose-ables are mainly relevant for maintaining stability as the focus of interest, when any agent, relative to their changing context, is being put under strain. Any increasing systemic strain calls for all attention and importance to be placed on keeping everything stable. ${ }^{21}$

The most rounded and socially open of the four catastrophe equations is the Butterfly Catastrophe, which has the stable choice outcomes (i.e. choose-ables) with all four coefficients coming into play. So the choose-ables are being scoped to take account of not only the agent's sense of own perspectives and preferences but also others' perspectives and preferences. The effect of nullifying the bias belief factor (i.e. $c=0$ ) in the Butterfly Catastrophe is telling as it tends to build a pocket of stability, ${ }^{22}$ which relates to forming compromises for resolution to complex social problems (sometimes referred to as "clumsy solutions" ${ }^{23}$ ). If the bias belief factor is allowed to increase (i.e. $c>0$ ), then this puts pressure on one side or the other of the pocket of stability until it disappears, with the effect of increasingly biased beliefs for the agent, which tends to destroy any compromises (along with its holding context).

The introduction of coefficients $c$ and $d$ into agents' choice-making would appear to provide a more socially based, human-like approach for agent-based models. Being able to describe and discuss such approaches for decision agent modelling would seem to be more appropriate for today's increasingly socially complex world. In addition, sharing of beliefs about plausible futures will help decision agents to avoid surprises and stalemating behaviour. Sharing of perspectives on what matters to other agents, and to society as a whole, helps agents to work towards choose-ables from a more holistic perspective of a shared purpose that can lead more readily to coherence in decision-making.

\section{Conclusions}

This paper re-introduces the term "choose-ables" because there does not seem to be a formal name for the concept of a choose-able; being the imagined deemed possible ways forward, that the agent has to construct, compose or create before they

\footnotetext{
${ }^{21}$ For example, the likelihood of an over-loaded ship capsizing can be described by a swallowtail equation. Geoffrey Vickers in his book "Freedom in a Rocking Boat: changing values in an unstable society" (1972) captures the essence of the difficulty here in trying to open-up the range of choose-ables, when the focus is on stabilising and normalising.

22 Christopher Zeeman puts it beautifully (with author's notes in Zeeman (1977): "In applications concerning the emergence of compromise, the butterfly factor $[d]$ will increase with time (if held and supported by agent in context); at first the compromise is fragile, in the sense that its stability is broken by any perturbation across the nearby sides of the pocket; but as the pocket grows in size the compromise becomes stronger, in the sense of being stable under increasingly large perturbations.".

23 Thompson (2008) refers to these as "clumsy solutions".
} 
can begin to choose. The important point being that the nature of the deeming is subjective: according to that agent at that time and in terms of their particular circumstances. This paper addresses what, in terms of human, organic, social, organisational, institutional constructs, might affect, adjust, adapt or shape an agent's choose-ables.

Two foundation theories are presented, Catastrophe theory and Culture theory, that combine to provide a way to describe human, social, institutional, organisational changes as a kind of dynamic landscape, over which an agent might be working and adapting to sustain themselves, to maintain appropriate levels of comfort, stability and balance, and to be in harmony with others. The constructs draw together the mathematical basis for discontinuity and non-linearity, as proposed by Catastrophe theory, with recent developments in Culture theory. Indeed, clumsy solutions from Culture theory and the Butterfly Catastrophe are descriptions of the same phenomenon. These are also linked to the subjectivity of choose-ables, as proposed and developed by Shackle in his imaginative and original studies on economic choice.

Two practical triangular constructs are offered to aid access to an agent's implicit rationale for choice. The triangular constructs help to discuss degrees of freedom for choice and to understand possible rationales for an agent's state of closed-ness or openness to ideas and of having enough capability 'bandwidth' in terms of the scope and nature of their choose-ables. Their changing conditions, when working within an institutional or organisational structure, or cultural set of norms, would naturally affect the choose-able bandwidth.

The ideas put forward in this paper offer a useful support to Drama theory and a practical addition to Decision theory and Game theory, as they provide ways to take into account the broader context, as changing conditions tend to make an agent adapt and adjust their choose-ables. The constructs help to relate the difficult subjective concepts of subordination, rules, norms, taboos, beliefs, myths, biases, preferences, focus of attention, and provide insights into the ways in which these might affect and shape an agent's choose-ables. The influence of any cultural relationships on an agent's adherence to, or blindness within, such a cultural context may then also be more readily understood and more openly appreciated. The simple constructs can help to work through questions to uncover assumptions about an agent's rationale and what may have brought them to a dilemma or to carry out a specific action. For example, why might an agent be assumed to have only that set of options? Can we imagine ourselves in their shoes (and in their heads) so that we can begin to ask questions and support an appreciative inquiry? The simple constructs offer a place to start to open up the dialogue, upon which other methods and techniques can then build.

So, theories of choice-making need not be limited to the analysis of option selection, set within an objective, psychological context. This paper provides ways of opening out the study of an agent's choice-making by imagining and conceptualising their rationales for trading-in or trading-out their choose-ables according to their social, personal, organizational, institutional and cultural contexts, and their openness (or otherwise) to adapt to their changing circumstances.

In summary, the constructs suggested here offer initial ways to support an appreciative inquiry about an agent's relational context and may help to understand why 
their choose-ables may be limited. This limited degree of freedom for choice can in turn limit an agent's scope of view and openness of mind to others' viewpoints and perspectives. The drawing together of Catastrophe theory and Culture theory offers new ways of seeing and imagining the shaping effects of relational contexts on an agent's choose-ables that then act as a medium through which agents are drawn to make choices and carry out observable actions. The non-linear mathematics does not and cannot offer objective solutions to problems. As in Shackle, the unknown variable " $x$ " is subjective and the equations capture openness to others' preferences, beliefs and perspectives.

The strength of the combination of the two theories lies in their descriptive power of subjective, relational concepts that hitherto have tended to remain hidden and tacit. Being able to describe and discuss these concepts in decision agent modelling would seem to be helpful and appropriate for today's increasingly socially complex world. In addition, sharing of beliefs about plausible futures will help decision agents to avoid surprises and stalemating behaviour. Sharing of perspectives on what matters to other agents, and to society as a whole, should help agents to work towards choose-ables from a more holistic perspective of a shared purpose that could lead more readily to coherence in decision-making.

Acknowledgements Thanks are due to many colleagues with whom I have collaborated while thinking about and developing the ideas in this paper; in particular, S Anders Christensson of the Swedish National Defence College who contributed many of the additional notes and pointed me to key references. Acknowledgements also to previous co-authors whose ideas and discussions have helped to shape the narrative: Professor Gillian Stamp, BIOSS Foundation; Professor Jim Q. Smith, Warwick University; Geoff Markham, Anthony Alston, Dr. David Marsay and Paddy Turner, QinetiQ; Jeremy Hilton, Cranfield University; Dr. Anne-Marie Grisogono, DSTO Australia; Patrick Beautement, Abaci partnership; General Sir Rupert Smith, British Army and Professor Gwythian Prins.

Open Access This article is distributed under the terms of the Creative Commons Attribution 4.0 International License (http://creativecommons.org/licenses/by/4.0/), which permits unrestricted use, distribution, and reproduction in any medium, provided you give appropriate credit to the original author(s) and the source, provide a link to the Creative Commons license, and indicate if changes were made.

\section{References}

Angyal A (1958) Foundations for a science of personality. Harvard University Press, Cambridge Arendt H (1982) Lectures on Kant's political philosophy. Harvester Press, Brighton

Balke T, Gilbert N (2014) How do agents make decision? A survey. J Artif Soc Soc Simul 17(4):13

Bennett P, Bryant J, Howard N (2001) Drama theory and confrontation analysis. In: Rosenhead J, Mingers J (eds) Rational analysis for a problematic world revisited. Wiley, Chichester, pp 225-248

Bradshaw JM (2003) Making agents acceptable to people. In: Mařík V, Pěchouček M, Müller J (eds) Multi-agent systems and applications III. CEEMAS 2003. Lecture notes in computer science, vol 2691. Springer, Berlin

Bradshaw JM, Jung H, Kulkarni S, Johnson M, Feltovich PJ, Allen J, Bunch L, Chambers N, Galescu L, Jeffers R, Suri N, Taysom W, Uszok A (2004a) Toward trustworthy adjustable autonomy in KAoS. https://www.researchgate.net/publication/221456608_Toward_Trustworthy_Adjustable_Auton omy_in_KAoS. Accessed June 2018

Bradshaw JM, Feltovitch PJ, Jung H, Kulkarni S, Taysom W, Uszok A (2004b) Dimensions of adjustable autonomy and mixed-initiative interaction. In: Nickles M et al (eds) AUTONOMY LNAI 2969. Springer, Berlin, pp 17-39 
Christensson SA (2008) Instruction sets to use and test a transformation towards an agreed end nonfailing state. In: Proceedings of 13th international command and control research and technology symposium. http://dodccrp.org/events/13th_iccrts_2008/CD/html/tracks_frames.html. Accessed Sept 2017

Clausewitz C (1873) On war. David Campbell Publishers (1993), New York. ISBN-13: 978-1857151213

Dodd L (2011) A theory of choices: melding black swans, swallowtails and butterflies. In: Proceedings international conference of complex systems, Boston New England Complex Systems Institute, USA. http://necsi.edu/events/iccs2011/papers/91.pdf. Accessed Sept 2017

Dodd L, Markham G (2012) C2 agility, different models of change and reasoning with time. In: 17th ICCRTS. http://dodccrp.org/events/17th_iccrts_2012/post_conference/papers/014.pdf. Accessed Sept 2017

Dodd L, Markham G (2013) Orders of C2 agility and implications for information and decision-making. In: International command and control research technology symposium, Institute of Defense Analysis, Virginia. http://dodccrp.org/events/18th_iccrts_2013/post_conference/papers/028.pdf. Accessed Sept 2017

Dodd L, Stamp G, Prins G (2008) Going from closed to open: how may we make it bearable? In: Proceedings international conference of complex systems, Boston New England Complex Systems Institute, USA. http://necsi.edu/events/iccs7/viewabstract.php?id=95. Accessed Sept 2017

Douglas M (2008) A history of grid-group culture theory. http://projects.chass.utoronto.ca/semiotics/ cyber/douglas1.pdf. Accessed Sept 2017

Douglas M, Wildavsky A (1982) Risk and culture: an essay on the selection of technical and environmental dangers. University of California Press, Berkeley

Emery FE, Trist EL (1963) The causal texture of organizational environments. In: XVII international congress of psychology, Washington, D.C., USA, 20-26 August 1963

Gibson JJ (1977) The theory of affordances. In: Shaw R, Bransford J (eds) Perceiving, acting, and knowing. Wiley, Hoboken, NJ

Gibson JJ (1979) The ecological approach to visual perception. Houghton Mifflin Company, Boston

Grisogono AM (2004) A generic framework for generating and evaluating C2 concepts. In: 9th ICCRTS, Copenhagen. http://dodccrp.org/events/9th_ICCRTS/CD/papers/034.pdf. Accessed Sept 2017

Howard N (1999) Confrontation analysis: how to win operations other than war. CCRP Publications, Washington D.C. http://www.dodccrp.org/files/Howard_Confrontation.pdf. Accessed Sept 2017

Khanna T (2014) Contextual intelligence, vol 92, no 9. Harvard Business Review, Boston, pp 58-68

Klugl F (2015) Affordance-based interaction design for agent-based simulation models. In: Bulling N (ed) 2015 Proceedings of the 12th European conference on multi-agent systems (EUMAS) Prague, December 2014

Marsay D (2015) Decision-making under radical uncertainty: an interpretation of Keynes' treatise on probability, economics e-journal special issue on radical uncertainty and its implications for economics. http://www.economics-ejournal.org/economics/journalarticles/2016-1. Accessed Sept 2017

Milner M (1986) A life of one's own. Virago, London

Norman DA (1999) Affordance, conventions and design. Interactions 6(3):38-43

Shackle GLS (1976) Time and choice. Keynes lecture in economics. Proc Br Acad 62:309-329

Smith JQ, Dodd L (2012) Regulating autonomous agents facing conflicting objectives: a command and control example. Decis Anal 9(2):165-171

Smith JQ, Harrison PJ, Zeeman EC (1981) The analysis of some discontinuous decision processes. Eur J Oper Res 7(1):30-43

Sylvan DA, Voss JF (1998) Problem representation in foreign policy decision making. Cambridge University Press, Cambridge. ISBN: 0-521-62293-X

Thompson M (1982) A three dimensional model. In: Douglas Mary (ed) Essays in the sociology of perception. Routledge \& Kegan Paul, London

Thompson M (2008) Organising and disorganising: a dynamic and non-linear theory of institutional emergence and its implications. Triarchy Press, Charmouth

Thompson M, Ellis R, Wildavsky A (1990) Cultural theory. Westview Press, Boulder

Turing AM (1952) The chemical basis of morphogenesis. Philos Trans R Soc Lond 237(641):37-72

Wells AJ (2002) Gibson's affordances and Turing's theory of computation. LSE. http://eprints.1se. ac.uk/2606/1/Affordances_and_Computation_APA_style_(LSERO).pdf. Accessed Sept 2017

Zeeman EC (1977) Catastrophe theory: selected papers. Addison Wesley, Boston 